\title{
IMOBILIZAÇÃO DE PEROXIDASE DE RAIZ FORTE POR ENCAPSULAMENTO EM MICROESFERAS DE ALGINATO NA PRESENÇA DE LÍQUIDOS IÔNICOS IMIDAZÓLIOS
}

\author{
F. M. S. SANTOS ${ }^{1}$, R. M. C. DOS SANTOS ${ }^{1}$, M. N. MELO ${ }^{1}$, Á. S. LIMA ${ }^{1}$, H. M. \\ ALVAREZ $^{2}$, C. M. F. SOARES ${ }^{1}$ e A. T. FRICKS ${ }^{1}$ \\ ${ }^{1}$ Universidade Tiradentes, Instituto de Tecnologia e Pesquisa \\ ${ }^{2}$ Universidade Estadual de Feira de Santana, Departamento de Ciências Exatas \\ Email para contato: flaviabiotec@gmail.com
}

\begin{abstract}
RESUMO - O presente trabalho teve como objetivo avaliar o efeito da presença de líquido iônicos (LIs) baseados no cátion imidazol $\left(\left[\mathrm{C}_{4} \mathrm{mim}\right] \mathrm{TF}_{2} \mathrm{~N}\right),\left[\mathrm{C}_{4} \mathrm{mim}\right] \mathrm{BF}_{4}, \quad\left[\mathrm{C}_{4} \mathrm{mim}\right] \mathrm{PF}_{6}$, $\left[\mathrm{C}_{4} \mathrm{mim}\right] \mathrm{HSO}_{4}$ e $\left.\left[\mathrm{C}_{4} \mathrm{mim}\right] \mathrm{Ac}\right)$ no encapsulamento de peroxidase de raiz forte (PRF) em microesferas de alginato. A metodologia envolve a incorporação da enzima em solução aquosa de alginato de sódio a $0,5 \%(\mathrm{~m} / \mathrm{v})$ seguida de gotejamento em solução de cloreto de cálcio $1 \mathrm{M}$. A influência do carregamento da enzima foi estudada na faixa de $0,08-0,65 \mathrm{mg}$ $\mathrm{PRF} / \mathrm{g}$ alginato. Os resultados mostram que o uso de $\left[\mathrm{C}_{4} \mathrm{mim}\right] \mathrm{BF}_{4}$ como aditivo neste processo de imobilização na concentração de $1 \%(\mathrm{~m} / \mathrm{v})$ promove um aumento significativo na recuperação de atividade (RA) da enzima (5,2 \% para 23,5\%). A utilização de LIs como aditivos no encapsulamento de PRF em microesferas de alginato apresenta efeito positivo na atividade da enzima encapsulada neste suporte.
\end{abstract}

\section{INTRODUÇÃO}

As enzimas são consideradas "catalisadores ambientalmente amigáveis" por suas características operacionais convergirem às exigências legais para que as indústrias operem seus processos em condições de desenvolvimento sustentável, ou seja, por meio da chamada "química verde" ou sistemas de tecnologia limpa. Tais características abrangem um vasto leque de aplicações para as enzimas em diversos setores industriais, tais como: alimentos, cosméticos e químico-farmacêutica.

A capacidade de peroxidases vegetais, tal como peroxidase de raiz forte (PRF), em catalisar a oxidação de compostos fenólicos torna estas enzimas aplicáveis no setor ambiental para monitoramento de contaminantes em efluentes provenientes das indústrias. A PRF é também aplicada na identificação de compostos antioxidantes em alimentos e bebidas, formulação de kits de diagnósticos e na remoção de peróxido de materiais, tais como alimentos e resíduos industriais. Peroxidases vegetais apresentam grandes vantagens também na indústria químico-farmacêutica, pois constitui um efetivo biocatalisador em reações de transferência seletiva de oxigênio.

Diante da vasta aplicabilidade das peroxidases, a imobilização da PRF é extremamente vantajosa e pode viabilizar sua utilização em processos oxidativos em geral. É importante ressaltar que enzimas imobilizadas são frequentemente, mais estáveis em temperaturas mais elevadas ou 
pHs diferenciados da condição ótima. Assim, o biocatalisador pode apresentar maior eficiência e tempo de meia vida durante as condições reacionais selecionadas para cada aplicação de interesse em diferentes processos industriais.

O principal interesse na imobilização de enzimas é obter um catalisador cuja atividade e estabilidade não sejam afetadas durante o processo, em comparação com sua forma livre. Em reações químicas e bioquímicas o uso de enzimas livres pode ser economicamente inviável e a recuperação do meio reacional pode ser muito complexa.

A obtenção de uma enzima imobilizada com atividade elevada está relacionada à utilização de suportes sólidos biocompatíveis, ou seja, devem tornar as enzimas acessíveis aos seus cofatores e substratos, quando aplicados em biotransformação. Merece destaque o uso de polímeros naturais como suporte na imobilização de enzimas, como alginato, carragenina, agarose, quitina, quitosana, por serem não tóxicos, biocompatíveis e biodegradáveis.

Na tentativa de melhorar a atividade catalítica tem sido relatado o uso de líquidos iônicos (LIs) como aditivos no processo de imobilização enzimática. A literatura mostra uma tendência de aumento nos estudos envolvendo a atividade e estabilidade das oxidoredutases na presença de LIs. Desta forma o objetivo deste trabalho foi imobilizar a peroxidase de raiz forte (PRF) em suporte de alginato pela metodologia de encapsulamento na ausência e presença de diferentes líquidos iônicos (LI) imidazólicos $\left(\mathrm{C}_{4}\right)$.

\section{MATERIAL E MÉTODOS}

A atividade enzimática foi determinada por método colorimétrico, baseado na mudança de absorvância a $470 \mathrm{~nm}$ devido à formação do produto de oxidação do guaiacol, o tetraguaiacol durante três minutos. O ensaio continha tampão fosfato de sódio $100 \mathrm{mM}(\mathrm{pH} 6,0) ; 0,04 \mathrm{~mL}$ do preparado enzimático (solução estoque de PRF: 0,32 $\mathrm{mg} / \mathrm{mL}$ ) diluído 200 vezes; $0,1 \mathrm{~mL}$ de solução de guaiacol $100 \mathrm{mM}$ e $0,1 \mathrm{~mL}$ de $\mathrm{H}_{2} \mathrm{O}_{2} 2,0 \mathrm{mM}$, a $25^{\circ} \mathrm{C}$. O volume total de reação: $3 \mathrm{~mL}$. Para dosagem de atividade das amostras imobilizadas, cerca de $8 \mathrm{mg}$ foram utilizados e foi feita a leitura pontual da absorvância em $470 \mathrm{~nm}$ após 3 minutos de reação.

Uma unidade de atividade enzimática (U) foi definida como a quantidade de enzima capaz de fornecer $1 \mu \mathrm{mol}$ de produto em $1 \mathrm{~min}$ a $25^{\circ} \mathrm{C}$, no $\mathrm{pH}$ específico para esta reação. $\varepsilon_{\text {tetraguaiacol: }}$ : $26,6 \mathrm{mM}^{-1} \mathrm{~cm}^{-1}$ (HIRATA et al., 1998).

A peroxidase de raiz forte foi imobilizada por encapsulamento em esferas de alginato de cálcio. Foi verificada a influência do carregamento da enzima na recuperação da atividade na faixa de $0,08-0,65 \mathrm{mgPRF} / \mathrm{g}$ suporte. Todos os experimentos foram realizados em triplicata.

A recuperação de atividade $(R A)$ foi determinada pelo número de unidades de atividade enzimática total presente no suporte $(U s)$ comparado às unidades de atividade enzimáticaoferecidas para imobilização (Uo), conforme Equação 1: 
Utilizando o carregamento mais adequado foram conduzidas as imobilizações de PRF na presença de LI $\left(\left[\mathrm{C}_{4} \mathrm{mim}\right] \mathrm{TF}_{2} \mathrm{~N} ;\left[\mathrm{C}_{4} \mathrm{mim}_{\mathrm{B}} \mathrm{BF}_{4} ;\left[\mathrm{C}_{4} \mathrm{mim}\right] \mathrm{HSO}_{4} ;\left[\mathrm{C}_{4} \mathrm{mim}\right] \mathrm{Ac}\right.\right.$ e $\left.\left[\mathrm{C}_{4} \mathrm{mim}\right] \mathrm{PF}_{6}\right)$, que foi simultaneamente adicionado com a enzima na proporção de $1 \%(\mathrm{~m} / \mathrm{v})$ com respeito ao volume total da reação de imobilização. A escolha da concentração de $1 \%(\mathrm{~m} / \mathrm{v})$ de LIs foi baseada em estudos prévios publicados pelo grupo de pesquisa (SOUZA et al., 2012 e 2013).

Para imobilizar a PRF por encapsulamento foi adotada metodologia descrita por Matto e Husain (2009): a enzima foi dissolvida na solução aquosa de alginato de sódio e gotejada $\mathrm{emCaCl}_{2}$ $1 \mathrm{M}$, mantida em agitação branda por aproximadamente $2 \mathrm{~h}$, formando as microesferas de alginato de cácio contendo PRF encapsulada. As microesferas foram lavadas com água destilada para a retirada do excesso de cloreto de cálcio e moléculas de enzimas não encapsuladas, em seguida foram secas em estufa a $40^{\circ} \mathrm{C}$ durante $2 \mathrm{~h}$.

\section{RESULTADOS E DISCUSSÃO}

\subsection{EFEITO DO CARREGAMENTO DA PRF}

A influência do carregamento da enzima foi estudada na faixa de 0,08-0,65 mgPRF/g de suporte para as três metodologias de imobilização selecionadas e os resultados são mostrados na Tabela 1. No encapsulamento, com carregamento de $0,08 \mathrm{mgPRF} / \mathrm{g}$ de suporte a RA foi de $6,58 \%$, embora quando levado em consideração o número de unidades imobilizadas, o carregamento de $0,16 \mathrm{mg} \mathrm{PRF} / \mathrm{g}$ de suporte mostrou-se mais eficiente $(0,19 \mathrm{U}$ imobilizadas $)$ mesmo sendo o rendimento de imobilização de 5,11 \% desta forma este foi o carregamento escolhido para os experimentos na presença de LIs.

No encapsulamento comumente nota-se a diminuição da atividade da enzima quando comparada a sua forma livre. Nos estudos realizados por Jamalet al., 2012; Matto e Hunsain 2009 e Alemzed e Nejatii 2009 também foi possível observar esse perfil, contudo quando realizado estudos de parâmetros operacionais como $\mathrm{pH}$, efeito da temperatura, agentes caotrópicos e reutilização foi possível observar estabilidade e maior resistência das enzimas utilizadas. Esses resultados mostram a capacidade das peroxidases imobilizadas por encapsulamento para utilização em bioprocessos de interesse químico-industrial.

O mecanismo proposto para microencapsulação da enzima é mostrado na Figura 1. O encapsulamento constitui uma boa metodologia de imobilização por proporcionar um meio ambiente aquoso relativamente inerte dentro da matriz, o que causa poucos danos à estrutura da enzima nativa. A microencapsulação em esferas alginato é considerada adequada para a imobilização de enzimas por apresentar vantagens como a natureza hidrofílica, presença de grupos carboxílicos, origem natural estabilidade mecânica e estabilidade em condições experimentais extremas (MATTO \& HUSAIN et al., 2009) . O esquema de encapsulamento da PRF em suporte de alginato está representado na Figura 1. 
Tabela 1. Estudo da influência do carregamento de PRF na imobilização por encapsulamento

\begin{tabular}{c|c}
$\begin{array}{c}\text { Carregamento } \\
\text { (mgPRF/g de suporte) }\end{array}$ & $\mathbf{U A}$ \\
0,65 & $(\mathbf{U})$ \\
\hline 0,32 & 12,79 \\
\hline 0,16 & 6,39 \\
0,08 & 2,94 \\
\hline
\end{tabular}

A .Total

(U)

0,21

0,16

0,19

0,10
RA

(\%)

$1,62 \pm 0,11$

$2,74 \pm 0,32$

$5,19 \pm 0,15$

$6,58 \pm 0,31$

UA - Unidades totais de atividade adicionada (U)

A. Total - (Atividade do biocatalisador imobilizado) X (massa seca do biocatalisador imobilizado)

RA - Recuperação de atividade (\%), calculada pela equação (1).

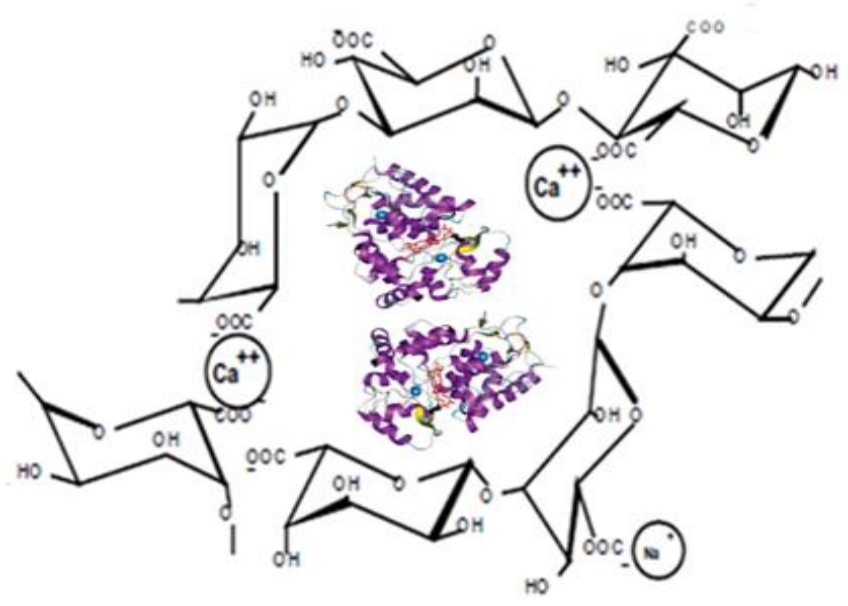

Figura 1.Esquema de imobilização da PRF nas microesferas de alginato de cálcio por encapsulamento.

\subsection{IMOBILIZAÇÃO DE PRF EM ALGINATO NA PRESENÇA DE LI}

Para a metodologia de encapsulamento a imobilização da PRF na presença de LIs $(1 \% \mathrm{~m} / \mathrm{v})$ foi conduzida utilizando carregamento de $0,16 \mathrm{mg}$ PRF/g suporte (2,9 U oferecidas). Pode-se observar que em todas as metodologias a presença de LI altera os valores de RA (Tabela 2).

Tabela 2. Estudo da influência de LIs na imobilização da HRP pela metodologia de encapsulamento.

\begin{tabular}{|c|c|c|c|c|c|}
\hline \multicolumn{6}{|c|}{$\mathrm{RA}(\%)$} \\
\hline $\begin{array}{l}\text { Ausência de } \\
\text { LI }\end{array}$ & {$\left[\mathrm{C}_{4} \mathrm{mim}\right] \mathrm{TF}_{2} \mathbf{N}$} & {$\left[\mathrm{C}_{4} \mathrm{mim}\right] \mathrm{BF}_{4}$} & {$\left[\mathrm{C}_{4} \mathrm{mim}\right] \mathrm{PF}_{6}$} & {$\left[\mathrm{C}_{4} \mathrm{mim}\right] \mathrm{Ac}$} & {$\left[\mathrm{C}_{4} \mathrm{mim}\right] \mathrm{HSO}_{4}$} \\
\hline $5,19 \pm 0,15$ & $20,4 \pm 3,4$ & $23,48 \pm 0,24$ & $17,32 \pm 0,17$ & $13,3 \pm 1,1$ & $8,9 \pm 0,15$ \\
\hline
\end{tabular}


Os resultados obtidos para os biocatalisadores imobilizados por encapsulamento mostram que o uso de LI como aditivo neste processo de imobilização promoveu um aumento de quatro vezes na recuperação de atividade quando comparado a imobilização na ausência de LIs. Os resultados relativos ao encapsulamento de PRF mostram que a RA foi de 23,5 e 20,40 \% na presença dos LIs $\left[\mathrm{C}_{4} \mathrm{mim}\right] \mathrm{BF}_{4} \mathrm{e}\left[\mathrm{C}_{4} \mathrm{mim}\right] \mathrm{Tf}_{2} \mathrm{~N}$, respectivamente. Na presença de $\left[\mathrm{C}_{4} \mathrm{mim}\right] \mathrm{Ac}$ a RA foi de $13,3 \%$, enquanto com $\left[\mathrm{C}_{4} \mathrm{mim}\right] \mathrm{PF}_{6}$ foi de $17,3 \%$. O $\left[\mathrm{C}_{4} \mathrm{mim}\right] \mathrm{HSO}_{4}$ mostrou o menor desempenho como aditivo entre os LIs estudados.

Estudos do grupo mostram que os resultados referentes à estabilidade de PRF na forma livre em meio aquoso contendo LIsimidazólios apresenta boa estabilidade, com exceção do $\left[\mathrm{C}_{4} \mathrm{mim}\right] \mathrm{HSO}_{4}$. Estes resultados sugerem que a presença de líquido iônico na imobilização da enzima por encapsulamento pode conduzir a modificações na estrutura das microesferas de alginato de cálcio que permitem um maior número de moléculas de enzima encapsuladas já que os LIs por si só não aumentam a atividade da enzima, como foi descrito por estudos previos realizados por este grupo de pesquisa.

A literatura relata que o F- dissociado do $\left[\mathrm{BF}_{4}\right]^{-}$e $\left[\mathrm{PF}_{6}\right]^{-}$gera um efeito negativo. PRF é uma heme proteína e seu sítio ativo é facilmente influenciado por F-. Sabe-se que LIs do tipo [BF $]^{-}$ pode dissociar $\mathrm{F}^{-}$em certas condições, o que pode afetar a atividade da PRF (LU et al., 2011). Contudo os resultados apresentados podem ser justificados pela natureza hidrofóbica dos LIs $\left[\mathrm{C}_{4} \mathrm{mim}\right] \mathrm{Tf}_{2} \mathrm{~N},\left[\mathrm{C}_{4} \mathrm{mim}\right] \mathrm{BF}_{4}$ e $\left[\mathrm{C}_{4} \mathrm{mim}\right] \mathrm{PF}_{6}$ que promove um aumento de moléculas de água ao redor da proteína, beneficiando a ação enzimática, devido ao aumento de moléculas de água livre. Esses LIs não tendem a formar ligações de hidrogênio, fato que ajuda a manter a estrutura conformacional da enzima (SOUZA et al., 2013, MARIE et al., 2010 e SINGH et al., 2010).

\section{CONCLUSÕES}

Os resultados do presente trabalho, que envolvem a imobilização de peroxidase de raiz forte em alginato de cálcio pela metodologia de encapsulamento permitem concluir que o carregamento ótimo para enzima imobilizada por este método foi $0,16 \mathrm{mgPRF} / \mathrm{g}$ de suporte. O uso de aditivo (LI imidazólico) durante o processo de síntese do suporte promoveu aumento significativo na RA, uma vez que, o encapsulamento mostrou RA de 5,19\% e 23,48\% na ausência e presença de LI $\left[\mathrm{C}_{4} \mathrm{mim}\right] \mathrm{BF}_{4}$, respectivamente. A presença de LI baseado em imidazólio na metodologia de encapsulamento validou a técnica de imobilização proporcionando um aumento de quatro vezes na recuperação da atividade.

\section{REFERÊNCIAS BIBLIOGRÁFICAS}

ALEMZADEH, I.; NEJATI, S. Phenols removal by immobilized horseradish peroxidase, Journal of Hazardous Materials, 166, p. 1082-1086, 2009.

HIRATA, T.; IZUMI, S.; OGURA, M.; YAWATA, T. Epoxidation of styrenes with the peroxidase from the culture cells of Nicotianatabacum, Tetrahedron, 54, p. 15993-16003, 1998.

JAMAL, F.; QIDWAI, T.; SINGH, D., PANDEY, P. K. Biocatalytic activity of immobilized pointed gourd (Trichosanthesdioica) peroxidase concanavalin. A complex on calcium alginate pectin gel, Journal of Molecular Catalysis B: Enzymatic, 74, p. 125- 131, 2012. 
MATTO, M; HUSAIN, Q. Calcium alginate-starch hybrid support for both surface immobilization and entrapment of bitter gourd (Momordicacharantia) peroxidase, Journal of Molecular Catalysis B: Enzymatic, 57, p. 164-170, 2009.

SOUZA, R. L., FARIA, E. L. P., FIGUEIREDO, R. T., FREITAS, L. S., IGLESIAS, M., MATTEDI, S., ZANIN, G.M., SANTOS, O. A. A., COUTINHO,J. A. P., LIMA, A. S., SOARES, C. M. F. Protic ionic liquid as additive on lipase immobilizationusing sol-gel, Enzymeand Microbial Technology, 52, p.141-150, 2013.

SOUZA, R. L.; RESENDE, W. C.; BARÃO, C. E.; ZANIN, G. M.; CASTRO, H. F.; SANTOS, O. A. A.; FRICKS, A. T.; FIGUEIREDO, R. T.; LIMA, A. S.; SOARES, C. M. F. Influence of the use of Aliquat 336 in the immobilization procedure in sol-gel of lipase from Bacillus sp. ITP-001, JournalMolecular Catalysis B: Enzymatic, 84, p. 152-159, 2012. 